Research Article

\title{
Calibration of Discrete Element Simulation Parameters for Powder Screw Conveying
}

\author{
Changpu Shen, Yongxiang Li, and Xuemeng Xu \\ College of Mechanical and Electrical Engineering, Henan University of Technology, Zhengzhou, Henan 450001, China \\ Correspondence should be addressed to Xuemeng Xu; 353882411@qq.com
}

Received 21 November 2020; Accepted 9 April 2021; Published 23 April 2021

Academic Editor: Yuanxin Zhou

Copyright (c) 2021 Changpu Shen et al. This is an open access article distributed under the Creative Commons Attribution License, which permits unrestricted use, distribution, and reproduction in any medium, provided the original work is properly cited.

In order to obtain the accurate contact parameters in the simulation process of powder screw conveying, this paper took wheat flour as an example, based on the discrete element JKR (Johnson-Kendall-Roberts) contact model, and directly calibrated the simulation contact parameters in the process of screw conveying in response to the mass flow rate of wheat flour. Firstly, the simulation density of wheat flour particles was calibrated, and the simulation density of wheat flour particles was $1320 \mathrm{~kg} / \mathrm{m}$. Then, Plackett-Burman experiment was used to screen out the parameters that had significant influence on the mass flow rate: surface energy JKR, coefficient of static friction between wheat flour and wheat flour, and the coefficient of static friction between wheat flour and stainless steel. The second-order regression model of mass flow rate and significance parameters was established and optimized based on Box-Behnken experiment, and the optimal combination of significance parameters: JKR was obtained to be 0.364; the static friction coefficient of wheat flour to wheat flour was 0.437 ; and the static friction coefficient of wheat flour to stainless steel was 0.609. Finally, the calibration parameters were used for simulation. By comparing the mass flow rate of simulation and experiment, the relative error of the two was $1.37 \%$. The simulation and experiment flow rate values at different rotating speeds $(60 \mathrm{r} / \mathrm{min}, 80 \mathrm{r} / \mathrm{min}, 100 \mathrm{r} / \mathrm{min}, 120 \mathrm{r} / \mathrm{min}$, and $140 \mathrm{r} / \mathrm{min})$ were further compared, and the errors were all within $3 \%$. The method of directly calibrating the simulation contact parameters through the screw conveying process can improve the accuracy of screw conveying simulation, and providing a method and basis for powder contact parameters calibration and screw conveying simulation of wheat flour.

\section{Introduction}

With small particle size and large specific surface area, the powder has good solubility, dispersibility, adsorbability, and chemical reactivity, etc. It is widely used in many fields such as chemical industry, medicine, food, electronics, and aviation. The powder is usually transported by screw conveyors when it is packaged and used as raw material. Experimental studies, theoretical analysis, and numerical simulation are usually used to study the screw conveying process of powder. Because the movement of powder particles in the screw conveyor is extremely complicated, it is difficult to describe the force and movement of powder particles through experimental research and theoretical analysis. The numerical simulation can make up for the deficiency of experimental research and theoretical analysis and can analyze the force and movement of powder in the process of screw conveying in detail. Moreover, it can also determine the effect of specific parameters on performance through sensitivity analysis and experimental detail reproduction.

Since the discrete element method was proposed by Cundall and Strack in 1980 [1], it has been used as an effective analytical method to study the structure and motion law of discontinuous particulate matter. In recent years, the gradually mature discrete element method (DEM) and relevant numerical analysis software can track and analyze the movement of particles very well in multi-scale structures and can greatly reduce the cost of bulk material research. Discrete element method has been widely used in agriculture [2-8], pharmaceutical [9], mining [10-12], steel manufacturing [13], and other industries. When the discrete element method or the corresponding numerical analysis 
software is used to analyze, the built particle model needs to define its physical parameters, mainly including intrinsic parameters (density, elastic modulus, Poisson's ratio), and parameters (static friction coefficient, crash recovery coefficient, rolling friction coefficient) between particles and particles, and particles and equipment. However, due to the different physical parameters of various material particles, it is necessary to calibrate the material particles before the numerical simulation. At present, a variety of materials have been calibrated at home and abroad, such as soybean [14, 15], corn $[16-18]$, wheat $[19,20]$, rice $[21,22]$, soil $[23,24]$, and coal $[25,26]$, etc., but the parameters of these materials are calibrated through the angle of repose, rather than directly through the equipment under normal working conditions. Due to the fluidity, adhesion, compressibility, and other characteristics of the bulk materials, the parameters of the materials are calibrated only through the angle of repose. When the parameters calibrated by this method are simulated, the numerical simulation error will be large. Using the discrete element parameters calibrated by angle of repose to simulate the screw conveying [27], the relative error of the mass flow rate in the simulation and experiment is up to $8.5 \%$. If the corresponding equipment is used to directly calibrate the material parameters, the error of numerical simulation caused by parameter calibration can be greatly reduced.

In this paper, wheat flour is used as the transport material; considering the cohesion between wheat flour particles, JKR contact model is adopted. On this basis, the simulation density of wheat flour particles was calibrated by experiment and numerical simulation firstly, and then the discrete element simulation contact parameters of wheat flour particles were calibrated by Plackett-Burman, steepest climb, and BoxBehnken experiments to obtain the optimal parameter combination. Finally, the optimal parameter combination is used to conduct the numerical simulation of the screw conveying at different speeds, and the mass flow rate of the numerical simulation is compared with the mass flow rate of the experiment, and the error is analyzed.

\section{JKR Contact Model}

In this paper, wheat flour was used as the material transported by screw. The particle size was small and contained $13 \%-15 \%$ water. There were van der Waals force, liquid bridge force, and electrostatic force among the particles, which made the particle surface cohesive. Hertz contact theory only considers the elastic deformation of particle interaction, but does not consider the surface adhesion between particles. In this paper, JKR contact model is adopted to consider the adhesion between particles, and surface energy is introduced to represent the adhesion between particles.

In the conveying process of wheat flour, the force between wheat flour particles is composed of normal force and tangential force, and the normal force is divided into normal damping force and normal elastic force, and the tangential force is divided into tangential damping force and tangential elastic force. The JKR contact physical model of the interaction between the two particles is shown in Figure 1.
JKR contact theory is an extension of Hertz contact theory. Based on Hertz contact theory, the interaction between two particles is analyzed as follows:

(1) Tangential damping force of two particles based on JKR model $F_{\tau}^{d}$ is

$$
F_{\tau}^{d}=-2 \sqrt{\frac{5}{6} c_{\tau}} \sqrt{k_{\tau} m^{*}} v_{\tau}^{\mathrm{rel}} .
$$

Here, $c_{\tau}, k_{\tau}, m^{*}$, and $v_{\tau}^{\overline{\mathrm{rel}}}$ represent the tangential damping coefficient, the tangential stiffness coefficient, the equivalent mass, and the tangential component of the relative velocity of the contact particle, respectively.

Among them, the damping coefficient $c_{\tau}$ is

$$
c_{\tau}=\frac{\ln e}{\sqrt{\ln ^{2} e+\pi^{2}}} .
$$

Here, $e$ and $\pi$ represent the particle recovery coefficient and the circumference ratio, respectively.

Tangential stiffness $k_{\tau}$ is

$$
k_{\tau}=8 G^{*} \sqrt{R^{*} \alpha} .
$$

Here, $G^{*}, R^{*}$, and $\alpha$ represent the particle equivalent shear modulus, the particle equivalent radius, and the particle normal overlap, respectively.

The equivalent shear modulus $G^{*}$ is

$$
G^{*}=\frac{2-v_{j}^{2}}{G_{i}}+\frac{2-v_{j}^{2}}{G_{j}} \text {. }
$$

Here, $v_{i}, v_{j}, G_{i}$, and $G_{j}$ represent the velocity of particle $i$, the velocity of particle $j$, the shear modulus of grain $i$, and the shear modulus of particle $j$, respectively.

Equivalent particle radius $R^{*}$ is

$$
\frac{1}{R^{*}}=\frac{1}{R_{i}}+\frac{1}{R_{j}} .
$$

Here, $R_{i}$ and $R_{j}$ represent the radius of particle $i$ and the radius of particle $j$, respectively.

Equivalent particle mass $m^{*}$ is

$$
\frac{1}{m^{*}}=\frac{1}{m_{i}}+\frac{1}{m_{j}} \text {. }
$$

Here, $m_{i}$ and $m_{j}$ represent the mass of particle $i$ and the mass of particle $j$, respectively.

(2) Tangential elastic force of two particles based on JKR model $F_{\tau}$ is

$$
F_{\tau}=k_{\tau} \delta_{\tau} .
$$

Here, $\delta_{\tau}$ and $k_{\tau}$ represent the particle tangential overlap and the average value, respectively. 


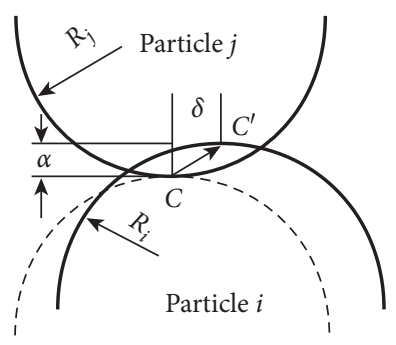

(a)

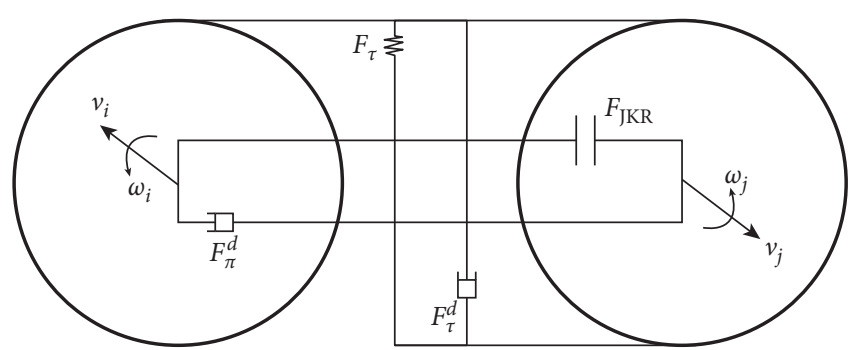

(b)

FIGURE 1: JKR physical model. (a) JKR contact model. (b) JKR contact force.

The tangential force is related to the friction force, and the friction force has a great influence on the simulation, so the static friction force $f$ is

$$
f=\mu_{s} F_{n}
$$

Here, $\mu_{s}$ and $F_{n}$ represent the coefficient of static friction and the normal force, respectively.

Rolling friction can be explained by the torque on the contact surface $T_{i}$ as

$$
T_{i}=-\mu_{r} R_{i} \omega_{i}
$$

Here, $\mu_{r}, R_{i}$, and $\omega_{i}$ represent the rolling friction coefficient, the radius of particle $i$, and the angular velocity of particle $i$, respectively.

(3) The normal damping force of two particles based on JKR model $F_{n}^{d}$ is

$$
F_{n}^{d}=-2 \sqrt{\frac{5}{6} c_{n}} \sqrt{k_{n} m^{*}} v_{n}^{\mathrm{rel}}
$$

Here, $c_{n}, k_{n}, m^{*}$, and $v_{n}^{\overline{\text { rel }}}$ represent the normal damping coefficient, the normal stiffness coefficient, the equivalent mass, and the tangential component of the relative, respectively.

Among them, the normal stiffness $k_{n}$ is

$$
k_{n}=8 E^{*} \sqrt{R^{*} \alpha}
$$

Here, $E^{*}, R^{*}$, and $\alpha$ represent the particle equivalent elastic modulus, the particle equivalent radius, and the particle normal overlap, respectively.

Equivalent modulus of elasticity $E^{*}$ is

$$
\frac{1}{E^{*}}=\frac{1-v_{j}^{2}}{E_{i}}+\frac{1-v_{j}^{2}}{E_{j}} .
$$

Here, $v_{i}, v_{j}, E_{i}$, and $E_{j}$ represent the velocity of particle $i$, the velocity of particle $j$, the elastic modulus of particle $i$, and the elastic modulus of particle $j$, respectively.

(4) In the JKR model, the normal phase elasticity can effectively reflect the viscoelastic characteristics between particles, so the normal phase elastic force $F_{\text {JKR }}$ is

$$
F_{\mathrm{JKR}}=-4 \sqrt{\pi \gamma E^{*}} \alpha^{3 / 2}+\frac{4 E^{*}}{3 R^{*}} \alpha^{3}
$$

Here, $\pi, \gamma, \alpha, E^{*}$, and $R^{*}$ represent the circumference ratio, the surface energy, the particle normal overlap, the particle equivalent elastic modulus, and the particle equivalent radius, respectively.

\section{Discrete Element Simulation Parameter Calibration}

3.1. Particle Density Calibration of Wheat Flour. Particle density is an essential and critical parameter in discrete element simulation of powder. However, it is difficult to obtain accurate particle density through experiments and there is a large error in simulation. In this paper, in order to obtain the accurate particle density of discrete element simulation of wheat flour, the volume density of wheat flour was obtained through the experiment firstly, and then the particle density of wheat flour was adjusted through simulation, so that the volume density of wheat flour obtained through experiment was equal to that of the simulated particle, so as to obtain the particle density of wheat flour.

3.1.1. Experimental Measurement of Wheat Flour Particle. The particle density of wheat flour was measured with a container and an electronic scale. The container has a diameter of $0.1 \mathrm{~m}$, a height of $0.195 \mathrm{~m}$, and a volume of $0.00152 \mathrm{~m}^{3}$. The measurement steps of bulk density of wheat flour are shown in Figure 2:

(1) Weigh the empty container $m_{\mathrm{c}}$

(2) Free fall the wheat flour from the entrance into the container until it overflows

(3) Scrape off the excess wheat flour and weigh the total $m_{\mathrm{t}}$

(4) According to $\rho_{c}=\left(m_{t}-m_{c} / V_{c}\right)$, measured wheat flour bulk density $\rho_{c}=500.13 \mathrm{~kg} / \mathrm{m}^{3}$

3.1.2. The Particle Density Calibration. The volume density of wheat flour was measured as $500.13 \mathrm{~kg} / \mathrm{m}^{3}$ through experiments. During the simulation process, the density of wheat flour simulated particles was adjusted to make its volume density as $500.13 \mathrm{~kg} / \mathrm{m}^{3}$. During the calibration of this density, a container with a radius of $0.1 \mathrm{~m}$ and a height of 


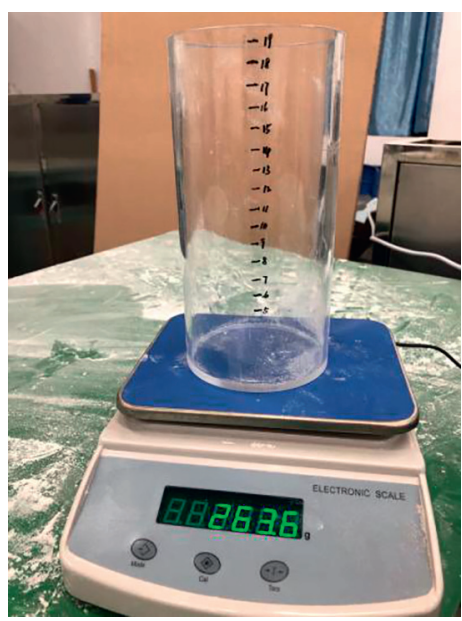

(a)

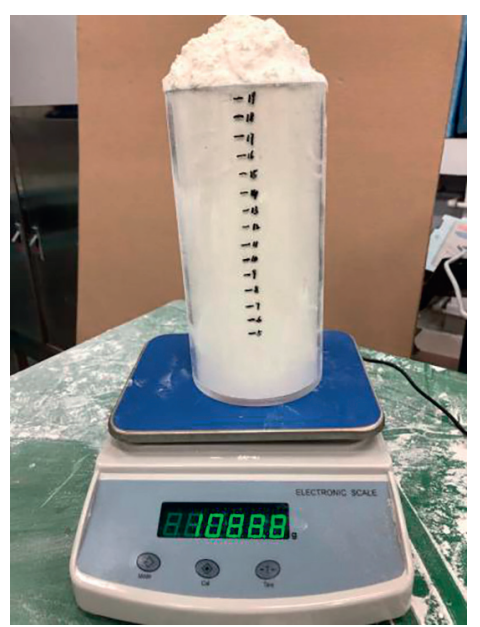

(b)

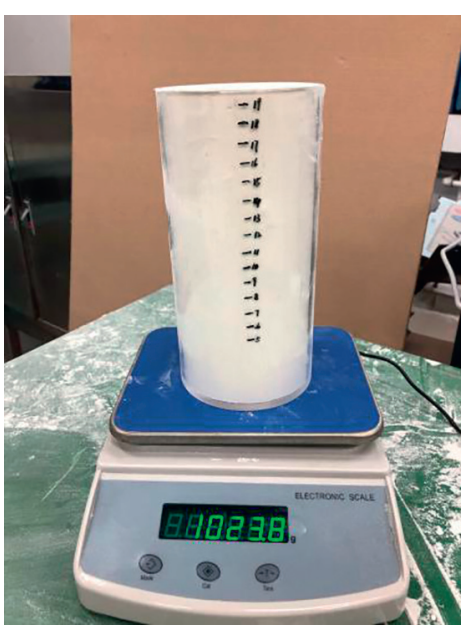

(c)

Figure 2: Measurement of wheat flour volume density. (a) Container weight measurement. (b) Pour the flour until it overflows. (c) Measurement of total weight of container and wheat flour.

$0.1 \mathrm{~m}$ was created, and a scraper was used to scrape off the excess simulation particles above the container. The calibration process of wheat grain density is shown in Figure 3.

Particle injection: particles are generated from the particle factory and fall freely into the container, so that particles fill the container and overflow.

Particles stand still: after the particles are filled, let them stand and freely overflow for a period of time to make the particles completely still.

Scrape particles: at the end of standing, the scraper moves to scrape off excess particles so that the particles in the container are flush with the mouth of the container.

Through the calibration of wheat flour simulated grain density, the grain density is $1320 \mathrm{~kg} / \mathrm{m}^{3}$.

\subsection{Calibration of Wheat Flour Particle Contact Parameters.} Using wheat flour flow rate as the response value, the discrete element simulation contact parameters of the wheat flour particles were calibrated directly in the screw conveying process by Plackett-Burman, steepest climbing, and Box-Behnken experiments, and the optimal combination of contact parameters was obtained. The discrete element simulation of wheat flour and other powders provides a reference.

\subsubsection{Mass Flow Rate Experiment of Wheat Flour. In this} experiment, the screw conveyor of Jingu Co., Ltd. was used. The pitch is $0.1 \mathrm{~m}$; the inner shaft diameter is $0.035 \mathrm{~m}$; the outer diameter of the blade is $0.12 \mathrm{~m}$; the inner diameter of the shaft cylinder is $0.13 \mathrm{~m}$; the gap is $0.005 \mathrm{~m}$; and the length of the feeding section is $0.5 \mathrm{~m}$; and speed range is $60 \mathrm{r} /$ $\mathrm{min}-150 \mathrm{r} / \mathrm{min}$. The rotation speed of this screw conveyor is $100 \mathrm{rad} / \mathrm{s}$. The weight of wheat flour was measured using a load cell (RS485 plane load cell: Hengyuan Sensor Technology Co., Ltd.; weighing range $0-50 \mathrm{~kg}$; sampling frequency $10-30 \mathrm{~Hz}$; measurement error $\pm 0.003 \mathrm{~kg}$ ). The data is set to record data every $0.1 \mathrm{~s}$ and the duration is set to $20 \mathrm{~s}$. After the data recording is completed, the wheat flour time- quality data is derived to calculate the mass flow rate of wheat flour. The mass flow rate value of the wheat flour was experimented 5 times to take the average value. The mass flow rate value of the wheat flour screw conveyor was measured to be $0.805 \mathrm{~kg} / \mathrm{s}$. The experimental process and time-mass curve are shown in Figure 4.

\subsubsection{Simulation Model and Parameters}

(1) Simulation parameters. Based on the settings of powder particle and stainless steel discrete element simulation parameters at home and abroad [28-32] and the built-in GEMM database of EDEM software, the variation range of each simulation parameter in this study is shown in Table 1. Combining the literature related to powder simulation [33-35] and the theory of particle scaling, the intrinsic parameters required for the simulation are set to wheat flour density of $1320 \mathrm{~kg} / \mathrm{m}^{3}$, wheat flour Poisson's ratio of 0.25 , and wheat flour shear modulus of $6.0 \times 10^{7} \mathrm{~Pa}$. The contact parameters of the material vary greatly with the density, shape, and particle size of the material, which cannot be obtained by consulting the physical property manual or literature, and using virtual experiments for calibration.

(2) Simulation model. This simulation refers to the actual physical model and is appropriately simplified. The design dimensions of the hopper and spiral are the same as the actual physical model. Due to simulation conditions and time constraints, the particles radius was magnified two times, and the circle of the enlarged particles was set to $0.1 \mathrm{~mm}$. Spherical particles were used for the simulation [36-39]. The simulation model is shown in Figure 5. During the simulation, the contact model is "Hertz-Mindlin with JKR," the simulation time is $10 \mathrm{~s}$, the particle generation method is dynamic, the generation rate is set to $200,0000 / \mathrm{s}$, the number of generations is set to 4000000 , and after the generation is completed, the rotation speed of the screw shaft 


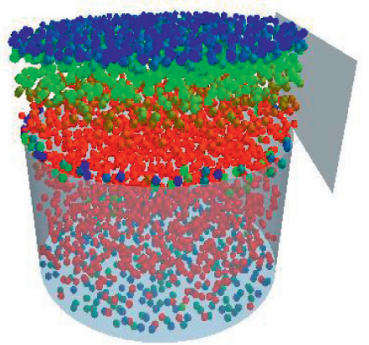

(a)

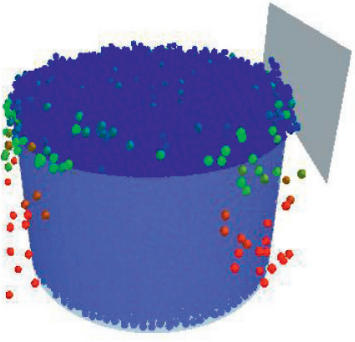

(b)

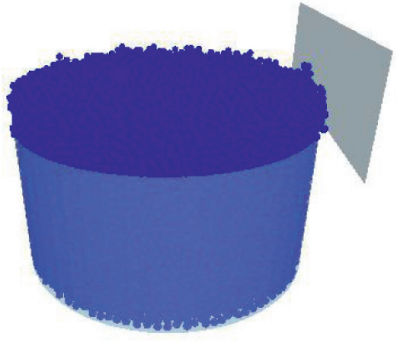

(c)

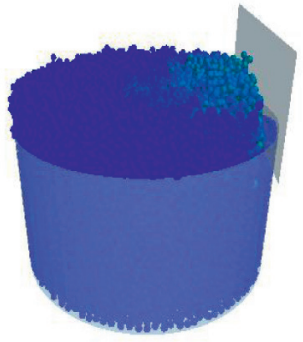

(d)

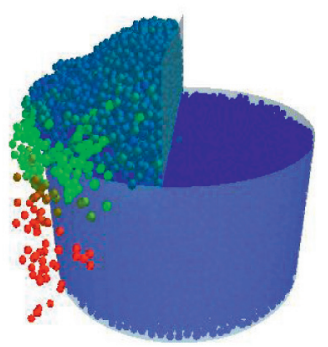

(e)

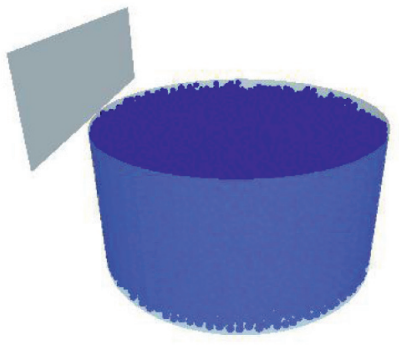

(f)

Figure 3: Calibration process of wheat flour simulated grain density. (a) Particles injection start. (b) Particles injection end. (c) Particles stand still. (d) Particles scraping begin. (e) Particles scraping process. (f) Particles scraping end.

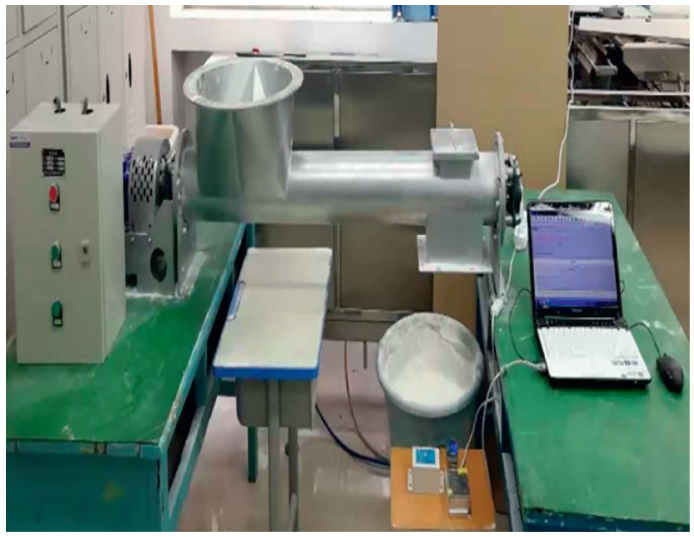

(a)

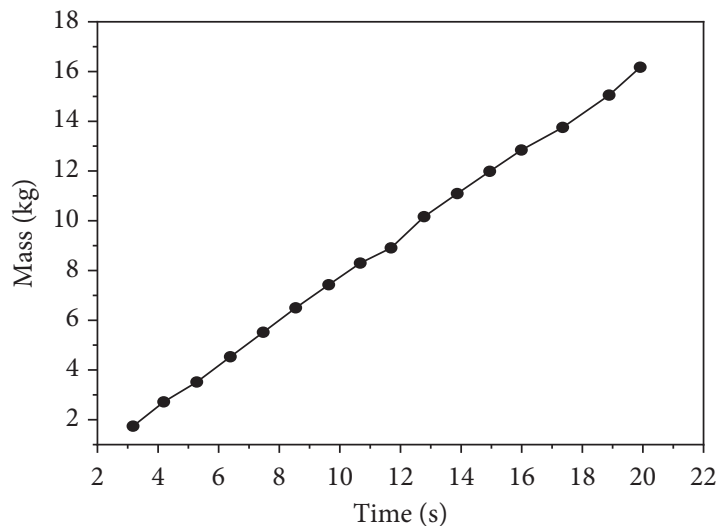

(b)

Figure 4: Determination of mass flow rate of wheat flour screw conveyor. (a) Mass flow rate experiment of wheat flour. (b) Time-mass curve

is set, and the rotation speed of the screw shaft is $100 \mathrm{rad} / \mathrm{s}$. After the simulation calculation is completed, the mass flow rate sensor in the software post-processing is used to measure the spiral conveyance of wheat flour.

\subsubsection{Design of Parameter Calibration Simulation Experiment}

(1) Plackett-Burman experiment. Plackett-Burman design is a two-level partial experimental design. Factor significance is determined by examining the relationship between the target response and each factor, and comparing the differences between the two levels of each factor. This PlackettBurman design screens the significance of simulated contact parameters based on the response rate of wheat spiral conveying mass flow rate. The low level is set to the original level, and the high level is set to twice the low level. The experiment parameters are shown in Table 2.

The design and results of Plackett-Burman are shown in Table 3. The analysis of the results by Design Expert software is used to obtain the significance of each contact parameter as shown in Table 4. From Table 4, it can be seen that JKR, the static friction coefficient of wheat flour-wheat flour, and the static friction coefficient of wheat flour-stainless steel $P<0.01$ have extremely significant effects on the spiral mass flow rate, while the remaining parameters $P>0.05$ have minimal effects on the spiral mass flow rate. In order to facilitate subsequent experiments, only the three parameters with the most significant impact $(P<0.01)$ are considered in the steepest climbing and Box-Behnken experiments. The rest of the parameters combined with the relevant literature $[20,23,24,34]$ take the 
TABle 1: Parameters required in DEM simulation.

\begin{tabular}{lc}
\hline Simulation parameters & Value \\
\hline Density of wheat flour $/\left(\mathrm{kg} \cdot \mathrm{m}^{-3}\right)$ & 1320 \\
Poisson's ratio of wheat flour & $0.2-0.4$ \\
Shear modulus of wheat $/ \mathrm{Pa}$ & $1.1 \times 107-6 \times 107$ \\
Density of stainless steel $/\left(\mathrm{kg} \cdot \mathrm{m}^{-3}\right)$ & 7800 \\
Poisson's ratio of stainless steel & 0.3 \\
Shear modulus of stainless steel/Pa & $7 \times 1010$ \\
Wheat flour-wheat flour restitution coefficient & $0.15-0.55$ \\
Wheat flour-wheat flour static friction coefficient & $0.2-0.8$ \\
Wheat flour-wheat flour rolling friction coefficient & $0.05-0.25$ \\
Wheat flour-stainless steel restitution coefficient & $0.15-0.55$ \\
Wheat flour-stainless steel static friction coefficient & $0.2-1.0$ \\
Wheat flour-stainless steel rolling friction coefficient & $0.1-0.8$ \\
JKR & $0.1-0.5$ \\
\hline
\end{tabular}

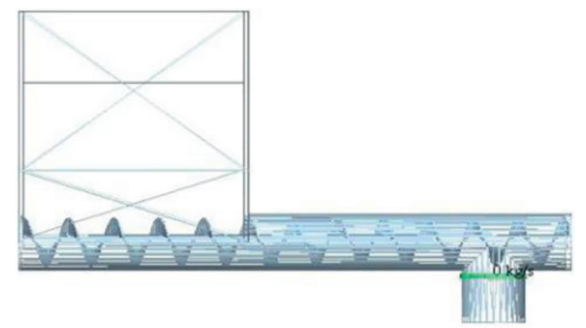

(a)

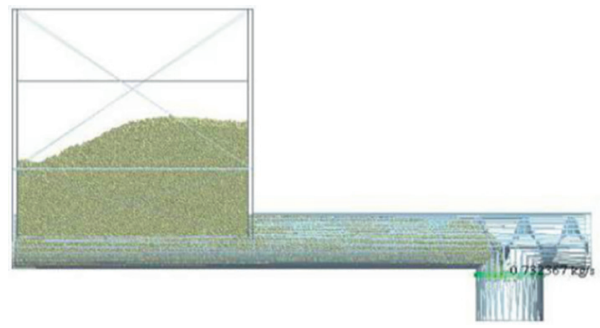

(b)

Figure 5: Simulation of wheat flour screw conveying. (a) Simulation process. (b). Post-processed mass flow rate.

TABle 2: Parameters of Plackett-Burman experiment.

\begin{tabular}{lccc}
\hline Symbol & Parameters & Low level & High level \\
\hline A & Wheat flour-wheat flour restitution coefficient & 0.15 & 0.3 \\
B & Wheat flour-wheat flour static friction coefficient & 0.2 & 0.05 \\
C & Wheat flour-wheat flour rolling friction coefficient & 0.15 & 0.1 \\
D & Wheat flour-stainless steel restitution coefficient & 0.2 & 0.3 \\
E & Wheat flour-stainless steel static friction coefficient & 0.1 & 0.4 \\
F & Wheat flour-stainless steel rolling friction coefficient & 0.1 & 0.2 \\
G & JKR & -1 & 0.5 \\
V1 & Virtual parameters 1 & -1 & 1 \\
V2 & Virtual parameters 2 & -1 & 1 \\
V3 & Virtual parameters 3 & -1 & 1 \\
V4 & Virtual parameters 4 & 1 \\
\hline
\end{tabular}

values (wheat flour-wheat flour recovery coefficient 0.2 , wheat flour-wheat flour rolling friction coefficient 0.15 , wheat flourstainless steel recovery coefficient 0.2 , and wheat flour-stainless steel rolling friction coefficient 0.15 ) for the steepest climbing and response surface experiment design.

(2) Steepest climbing experiment. After the Plackett-Burman experiment, the steepest climbing experiment is performed according to the screened significance parameters so that it can quickly enter the vicinity of the optimal value. The steepest climbing experiment starts from the center point of the PB experiment, and the climbing step length is determined according to the regression coefficient obtained from the $\mathrm{PB}$ experiment. In order to approach the optimal value as quickly as possible, the climbing step length is usually taken as a larger value. The selected steps and results of this climbing experiment are shown in Table 5. According to the results in Table 5, it can be seen that the relative error of the mass flow rate at the $3 \mathrm{rd}$ level is the smallest, and the relative error from the 2 nd to the 4 th level changes from large to small and then becomes larger. Therefore, the 3rd level is selected as the center point, and the 2nd and 4th levels are follow-up response surface design at low and high levels.

(3) Box-Behnken experiment and regression model. Based on the results of the steepest climbing experiment and the principle of response surface design, the low, medium, and high levels of significant parameters were selected for the 
Table 3: Design and results of Plackett-Burman experiment.

\begin{tabular}{|c|c|c|c|c|c|c|c|c|c|c|c|c|}
\hline Running sequence & $\mathrm{A}$ & $\mathrm{B}$ & $\mathrm{C}$ & $\mathrm{D}$ & $E$ & $\mathrm{~F}$ & $\mathrm{G}$ & V1 & $\mathrm{V} 2$ & V3 & $\mathrm{V} 4$ & Mass flow rate $(\mathrm{kg} / \mathrm{s})$ \\
\hline 1 & -1 & 1 & -1 & 1 & 1 & -1 & 1 & 1 & 1 & -1 & -1 & 0.8037 \\
\hline 2 & 1 & -1 & 1 & 1 & 1 & -1 & -1 & -1 & 1 & -1 & 1 & 1.1006 \\
\hline 3 & -1 & 1 & 1 & -1 & 1 & 1 & 1 & -1 & -1 & -1 & 1 & 0.8108 \\
\hline 4 & 1 & 1 & -1 & -1 & -1 & 1 & -1 & 1 & 1 & -1 & -1 & 1.1291 \\
\hline 5 & 1 & -1 & 1 & 1 & -1 & 1 & 1 & 1 & -1 & -1 & -1 & 1.1444 \\
\hline 6 & -1 & -1 & -1 & -1 & -1 & -1 & -1 & -1 & -1 & -1 & -1 & 1.119 \\
\hline 7 & 1 & 1 & -1 & 1 & 1 & 1 & -1 & -1 & -1 & 1 & -1 & 1.0036 \\
\hline 8 & 0 & 0 & 0 & 0 & 0 & 0 & 0 & 0 & 0 & 0 & 0 & 1.0242 \\
\hline 9 & 1 & 1 & 1 & -1 & -1 & -1 & 1 & -1 & 1 & 1 & -1 & 1.0007 \\
\hline 10 & 1 & -1 & -1 & -1 & 1 & -1 & 1 & 1 & -1 & 1 & 1 & 0.895 \\
\hline 11 & -1 & -1 & -1 & 1 & -1 & 1 & 1 & -1 & 1 & 1 & 1 & 1.0931 \\
\hline 12 & -1 & -1 & 1 & -1 & 1 & 1 & -1 & 1 & 1 & 1 & -1 & 1.0307 \\
\hline 13 & -1 & 1 & 1 & 1 & -1 & -1 & -1 & 1 & -1 & 1 & 1 & 1.032 \\
\hline
\end{tabular}

TABle 4: Analysis of the significance of parameters in Plackett-Burman experiment.

\begin{tabular}{lcccc}
\hline Parameters & Effect & Sum of squares & Contribution/\% & Significance \\
\hline A & 0.064 & 0.012 & 7.896 & 4 \\
B & -0.100 & 0.031 & 19.454 & 3 \\
C & 0.013 & 0.001 & 0.307 & 7 \\
D & 0.032 & 0.003 & 1.975 & 6 \\
E & -0.146 & 0.036 & 40.874 & 1 \\
F & 0.043 & 0.006 & 3.637 & 5 \\
G & -0.111 & 0.037 & 23.833 & 2 \\
\hline
\end{tabular}

TABLe 5: Design and results of steepest ascent experiment.

\begin{tabular}{cccccc}
\hline No. JKR & $\begin{array}{c}\text { Wheat flour-wheat flour rolling friction } \\
\text { coefficient }\end{array}$ & $\begin{array}{c}\text { Wheat flour-stainless steel static friction } \\
\text { coefficient }\end{array}$ & $\begin{array}{c}\text { Mass flow rate } \\
(\mathrm{kg} / \mathrm{s})\end{array}$ & $\begin{array}{c}\text { Relative error } \\
(\%)\end{array}$ \\
\hline 1 & 0.1 & 0.2 & 0.2 & 1.188 & 47.58 \\
2 & 0.2 & 0.4 & 0.4 & 0.961 & 19.38 \\
3 & 0.3 & 0.6 & 0.6 & 0.791 & 1.74 \\
4 & 0.4 & 0.8 & 0.8 & 0.749 & 6.96 \\
5 & 0.5 & 1.0 & 1.0 & 0.677 \\
\hline
\end{tabular}

experiment design, and three center points were selected to evaluate the error. The Box-Behnken experiment results are shown in Table 6. Using Design Expert to establish a secondorder regression equation with three significant parameters and the mass flow rate value is

$$
\begin{aligned}
Q= & 1.6+0.35 H-1.8 J-1.1 K+0.036 H J+0.088 H K \\
& -0.074 J K-0.452 H^{2}+1.153 J^{2}+1.538 K^{2} .
\end{aligned}
$$

Box-Behnken experiment model analysis results are shown in Table 7. According to the results in Table 7, the fitted model $P<0.005$; JKR surface energy $(\mathrm{H})$, static friction coefficient between wheat flour particles $(\mathrm{J})$, and static friction coefficient of wheat flour-stainless steel (K), the quadratic term of JKR surface energy $\left(\mathrm{H}^{2}\right)$, and the quadratic term of the static friction coefficient between wheat flour particles $\left(\mathrm{J}^{2}\right)$ are all less than 0.05 , indicating that each parameter has a significant effect on the mass flow rate, and the regression model is valid. The coefficient of variation $\mathrm{CV}=0.72 \%$ in the experiment, which indicates that the experiment has higher reliability. The determination coefficient $R^{2}=0.9828$; the adjusted determination coefficient Adjusted $R^{2}=0.9520$; it shows that the model can truly reflect the actual situation. The experiment precision is Adep Precision $=18.42$, which shows that the model has good accuracy.

According to the results in Table 8, under the premise of ensuring the model is good, remove the terms that have no significant effect on the mass flow rate. The results of the analysis of variance after optimizing the model are shown in Table 8 . The misfit term $P=0.0279$; the coefficient of variation $\mathrm{CV}=1.91 \%$; Coefficient $R_{2}=0.9712$; Adjusted $R^{2}=0.9552$; Predicted $R^{2}=0.9131$; experiment precision Adep Precision $=23.5$. It can be seen that the model has good fit, reliability, and accuracy, which has been improved to some extent before optimization. The regression equation after optimization is 
TABLe 6: Design and results of Box-Behnken experiment.

\begin{tabular}{|c|c|c|c|c|}
\hline $\begin{array}{l}\text { Running serial } \\
\text { number }\end{array}$ & $\begin{array}{c}\text { Wheat flour-wheat } \\
\text { static friction coefficient }(\mathrm{H})\end{array}$ & $\begin{array}{c}\text { Coefficient of static friction } \\
\text { of wheat flour-stainless steel }(\mathrm{J})\end{array}$ & JKR (K) & $\begin{array}{l}\text { Mass flow } \\
\text { rate }(\mathrm{kg} / \mathrm{s})\end{array}$ \\
\hline 1 & 0 & 0 & 0 & 0.7991 \\
\hline 2 & 0 & 0 & 0 & 0.805 \\
\hline 3 & -1 & 0 & -1 & 0.8381 \\
\hline 4 & 0 & -1 & -1 & 0.9718 \\
\hline 5 & 1 & 0 & -1 & 0.7952 \\
\hline 6 & 0 & 1 & 1 & 0.753 \\
\hline 7 & 0 & -1 & 1 & 0.9404 \\
\hline 8 & -1 & -1 & 0 & 0.9475 \\
\hline 9 & 0 & 1 & -1 & 0.7903 \\
\hline 10 & 0 & 0 & 0 & 0.803 \\
\hline 11 & 1 & 1 & 0 & 0.7162 \\
\hline 12 & -1 & 1 & 0 & 0.7925 \\
\hline 13 & 1 & -1 & 0 & 0.8655 \\
\hline 14 & -1 & 0 & 1 & 0.8006 \\
\hline 15 & 1 & 0 & 1 & 0.7648 \\
\hline
\end{tabular}

TABLE 7: ANOVA of quadratic polynomial model of Box-Behnken experiment.

\begin{tabular}{|c|c|c|c|c|c|}
\hline Source of variation & Mean square & Freedom & Quadratic sum & F value & $P$ value \\
\hline Model & 0.0063 & 9 & 0.0085 & 31.83 & 0.0007 \\
\hline $\mathrm{H}$ & 0.0070 & 1 & 0.0070 & 26.36 & 0.0037 \\
\hline $\mathrm{J}$ & 0.0566 & 1 & 0.0566 & 212.69 & $<0.0001$ \\
\hline K & 0.0023 & 1 & 0.0023 & 8.76 & 0.0315 \\
\hline $\mathrm{HJ}$ & $8.1 \times 10-6$ & 1 & $8.1 \times 10-6$ & 0.0305 & 0.8682 \\
\hline HK & 0.0000 & 1 & 0.0000 & 0.0473 & 0.8364 \\
\hline JK & $8.7 \times 10-6$ & 1 & $8.7 \times 10-6$ & 0.0327 & 0.8637 \\
\hline $\mathrm{H}^{2}$ & 0.0012 & 1 & 0.0012 & 4.53 & 0.0867 \\
\hline $\mathrm{J}^{2}$ & 0.0079 & 1 & 0.0079 & 29.50 & 0.0029 \\
\hline $\mathrm{K}^{2}$ & 0.0009 & 1 & 0.0009 & 3.28 & 0.1299 \\
\hline Residual & 0.0013 & 5 & 0.0003 & & \\
\hline Lack of fit & 0.0013 & 3 & 0.0004 & 48.64 & 0.0202 \\
\hline Pure error & 0.0000 & 2 & $9 \times 10-6$ & & \\
\hline Sum & 0.0776 & 14 & & & \\
\hline \multicolumn{6}{|c|}{$R^{2}=0.9828$. Adjusted $R^{2}=0.9520$. Predicted $R^{2}=0.7287 . \mathrm{CV}=0.72 \%$. Adep Precision $=18.42$} \\
\hline
\end{tabular}

TABLE 8: ANOVA of the modified model of Box-Behnken experiment.

\begin{tabular}{|c|c|c|c|c|c|}
\hline Source of variation & Mean square & Freedom & Quadratic sum & F Value & $P$ value \\
\hline Model & 0.0754 & 5 & 0.0151 & 60.73 & $<0.0001$ \\
\hline $\mathrm{H}$ & 0.0070 & 1 & 0.0070 & 28.28 & 0.0005 \\
\hline $\mathrm{J}$ & 0.0566 & 1 & 0.0566 & 228.17 & $<0.0001$ \\
\hline K & 0.0023 & 1 & 0.0023 & 9.39 & 0.0135 \\
\hline $\mathrm{H} 2$ & 0.0014 & 1 & 0.0014 & 5.55 & 0.0430 \\
\hline $\mathrm{J} 2$ & 0.0075 & 1 & 0.0075 & 30.22 & 0.0004 \\
\hline Residual & 0.0022 & 9 & 0.0002 & & \\
\hline Lack of fit & 0.0022 & 7 & 0.0003 & 35.17 & 0.0279 \\
\hline Pure error & 0.0000 & 2 & $9 \times 10-6$ & & \\
\hline Sum & 0.0776 & 14 & & & \\
\hline \multicolumn{6}{|c|}{$R^{2}=0.9712$. Adjusted $R^{2}=0.9552$. Predicted $R^{2}=0.9131 . \mathrm{CV}=1.9 \%$. Adep Precision $=23.5$} \\
\hline
\end{tabular}




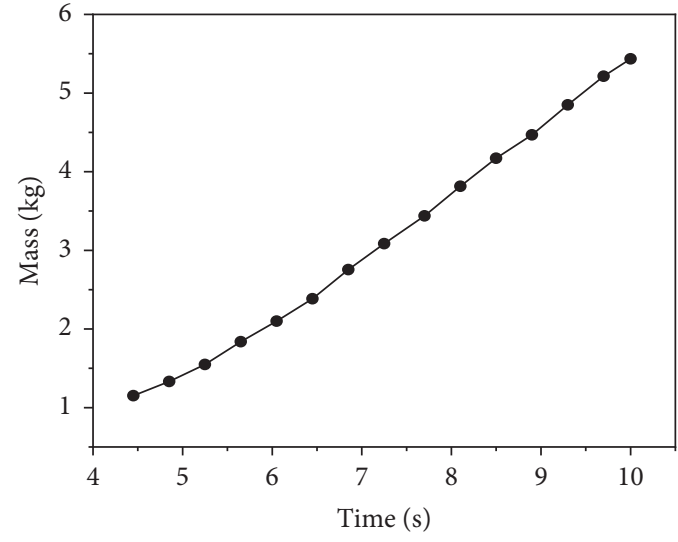

(a)

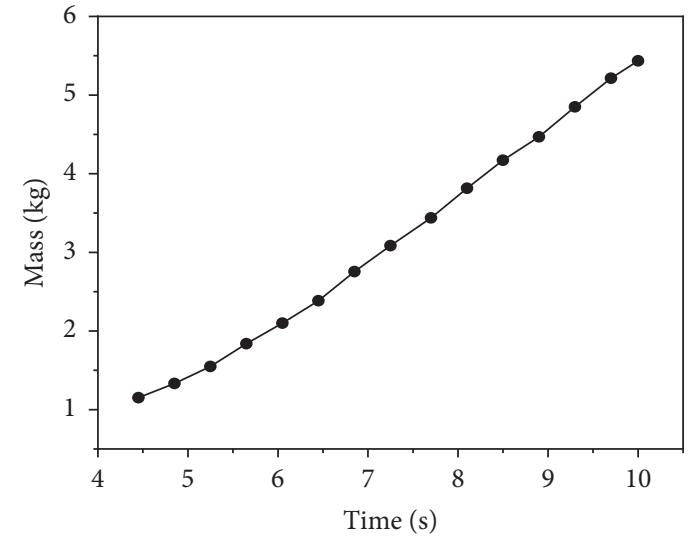

(b)

FIgURE 6: Comparison of simulation and physical experiments. (a) Simulation experiment. (b) Physical experiment.

$$
\begin{array}{r}
Q=1.435+0.429 H-1.769 J \\
-0.171 K-0.481 H^{2}+1.124 J^{2} .
\end{array}
$$

\section{Experiment Verification}

\subsection{Determination of the Best Parameter Combination and} Simulation Verification. Using Design Expert software to take the actual wheat flour mass flow rate as the target and optimize the regression equation after optimization, we can understand that, to minimize the mass flow rate error obtained by simulation and experiment, the surface energy of JKR is 0.364 and the static friction coefficient of wheat flourwheat flour is 0.437 , and the coefficient of static friction of wheat flour-stainless steel is 0.609 . The best parameter combination was used for the wheat flour mass flow rate simulation experiment. The comparison between the simulation and the physical experiment is shown in Figure 6. The mass flow rate value obtained from the simulation experiment was $0.816 \mathrm{~kg} / \mathrm{s}$, and the error value was $1.37 \%$ from the actual value of $0.805 \mathrm{~kg} / \mathrm{s}$, indicating that the simulation results were not significantly different from the actual experiment values.

4.2. Simulation Verification at Different Speeds. In order to further confirm the reliability of the contact parameters of wheat flour calibration, the simulation and experimental mass flow rates at different speeds are compared, as shown in Figure 7. It can be seen from the comparison that the mass flow rate errors at different speeds are all within 3\%, and the DEM simulation is very close to the experiment.

\section{Conclusion}

(1) Based on the JKR model in discrete element method, the simulated contact parameters required for the screw conveying of wheat flour were calibrated. The Plackett-Burman experiment screened out the factors that significantly affected the mass flow rate of screw conveying of wheat flour: the static friction

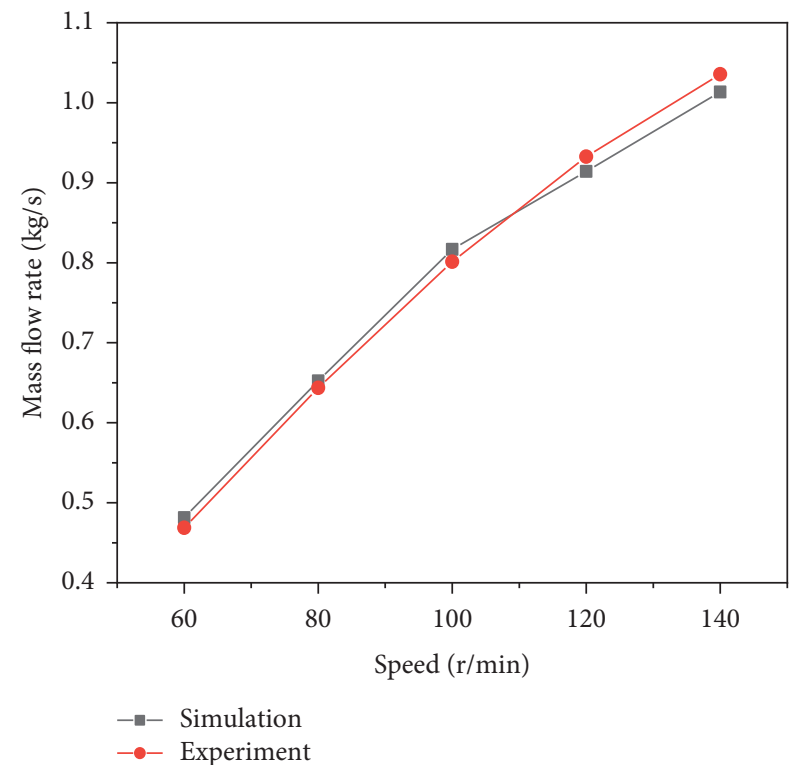

FIgURE 7: Comparative analysis of simulation and experiment of screw conveying at different speeds.

coefficient of wheat flour-wheat flour, the static friction coefficient of wheat flour-stainless steel, and surface energy JKR.

(2) Based on the results of the Box-Behnken experiment, a quadratic regression model between three significant parameters and mass flow rate was established and optimized. According to the results of the analysis of variance of the optimized model, it is known that, except for three significant parameters (JKR surface energy, wheat flour-wheat flour static friction in addition to the primary term of the coefficient, and the static friction coefficient of wheat flour-stainless steel), the quadratic term of the JKR surface energy $\left(\mathrm{H}^{2}\right)$ and the secondary term of the static friction coefficient of wheat flour-wheat flour (J2) also have a significant effect on the mass flow rate of screw flour transportation. 
(3) Taking the actual mass flow rate value of wheat flour screw conveying as the target, the regression equation was optimized and the best combination of significant parameters was obtained: wheat flourwheat static friction coefficient is 0.437 , wheat flourstainless steel static friction coefficient is 0.609 , and the surface energy JKR is 0.364 .

(4) Use the calibrated contact parameters for simulation, and compare the simulated mass flow rate with the experimental mass flow rate. The error value is only $1.37 \%$. To further demonstrate the accuracy of the calibration parameters, the simulation and experimental results at different speeds are compared. The mass flow rates were compared, and the error values were all in the range of $3 \%$. It is shown that parameter calibration in the process of screw conveying can improve the accuracy of the parameters and provide a reference for the screw conveying simulation of powder.

\section{Data Availability}

The main data are presented in the paper. More detailed data are available from the corresponding author upon request.

\section{Conflicts of Interest}

The authors declare that they have no conflicts of interest.

\section{Acknowledgments}

This work was supported by the National Key Research and Development Project of China (2018YFD0400704) and the Natural Science Project of Science and Technology Department of Henan Province (182102110163).

\section{References}

[1] P. A. Cundall and O. D. L. Strack, "Discussion: a discrete numerical model for granular assemblies," Géotechnique, vol. 30, no. 3, pp. 331-336, 1980.

[2] A. O. Raji and J. F. Favier, "Model for the deformation in agricultural and food particulate materials under bulk compressive loading using discrete element method. I: theory, model development and validation," Journal of Food Engineering, vol. 64, no. 3, pp. 359-371, 2004.

[3] K. G. Santos, A. V. P. Campos, O. S. Oliveira et al., "Barrozo m.a.s., dem simulations of dynamic angle of repose of acerola residue: a parametric study using a response surface technique," XX Congresso Brasileiro De Engenharia Química, vol. 1, pp. 1-8, 2015.

[4] F. Lv, X. Wang, M. Zhang, H. Fu, and J. Yu, Determination and Analysis for Parameters of Shape, Size, Physical and Mechanical Properties of Soybean Grains", International Conference on Discrete Element Methods, Springer, Singapore, 2017.

[5] X. Wen, H. Jia, S. Zhang et al., "Test of suspension velocity of granular fertilizer based on EDEM-fluent coupling," Transactions of the Chinese Society for Agricultural Machinery, vol. 51, no. 3, pp. 69-77, 2020.
[6] J. Chen, H. Zhou, Z. Zhao et al., "Analysis of rice seeds motion on vibrating plate using EDEM," Transactions of the Chinese Society for Agricultural Machinery, vol. 42, no. 10, pp. 79-83, 2020.

[7] Y. Han, F. Jia, Y. Zeng, L. Jiang, Y. Zhang, and B. Cao, "DEM study of particle conveying in a feed screw section of vertical rice mill," Powder Technology, vol. 311, no. 15, pp. 213-225, 2017.

[8] J. Horabik and M. Molenda, "Parameters and contact models for DEM simulations of agricultural granular materials: a review," Biosystems Engineering, vol. 147, pp. 206-225, 2016.

[9] W. R. Ketterhagen, M. T. am Ende, and B. C. Hancock, "Process modeling in the pharmaceutical industry using the discrete element method," Journal of Pharmaceutical Sciences, vol. 98, no. 2, pp. 442-470, 2009.

[10] S. Yang, W. Tian, and Y. Ju, "Discrete element simulation on shear deformation and crack evolution mechanism of coal specimen," Journal of China University of Mining \& Technology, vol. 45, pp. 1148-1155, 2016.

[11] G. W. Delaney, R. D. Morrison, M. D. Sinnott, S. Cummins, and P. W. Cleary, "DEM modelling of non-spherical particle breakage and flow in an industrial scale cone crusher," Minerals Engineering, vol. 74, pp. 112-122, 2015.

[12] B. A. Poulsen and D. P. Adhikary, "A numerical study of the scale effect in coal strength," International Journal of Rock Mechanics and Mining Sciences, vol. 63, pp. 62-71, 2013.

[13] H. Mio, M. Kadowaki, S. Matsuzaki, and K. Kunitomo, "Development of particle flow simulator in charging process of blast furnace by discrete element method," Minerals Engineering, vol. 33, no. 6, pp. 27-33, 2012.

[14] H. Jia, Y. Chen, J. Zhao et al., "Design and experiment of pneumatic-mechanical combined precision metering device for soybean," Transactions of the Chinese Society for Agricultural Machinery, vol. 49, no. 4, pp. 75-86, 2018.

[15] R. N. Cunha, K. G. Santos, R. N. Lima, C. R. Duarte, and M. A. S. Barrozo, "Repose angle of monoparticles and binary mixture: an experimental and simulation study," Powder Technology, vol. 303, pp. 203-211, 2016.

[16] D. Han, D. Zhang, L. Yang et al., "EDEM-CFD simulation and experiment of working performance of inside-filling airblowing seed metering device in maize," Transactions of the Chinese Society for Agricultural Machinery, vol. 33, no. 013, pp. 23-31, 2017.

[17] X. Wang, J. Yu, F. Lv, Y. Wang, and H. Fu, "A multi-sphere based modelling method for maize grain assemblies," Advanced Powder Technology, vol. 28, no. 2, pp. 584-595, 2017.

[18] L. Wang, R. Li, B. Wu, Z. Wu, and Z. Ding, "Determination of the coefficient of rolling friction of an irregularly shaped maize particle group using physical experiment and simulations," Particuology, vol. 38, pp. 185-195, 2018.

[19] Y. Liu, J. Zhang, and J. Chen, "Construction of visco-elastoplasticity contact model of vibratory screening and its parameters calibration for wheat," Transactions of the Chinese Society of Agricultural Engineering, vol. 34, no. 15, pp. 37-43, 2018.

[20] A. Patwa, R. P. K. Ambrose, and M. E. Casada, "Discrete element method as an approach to model the wheat milling process," Powder Technology, vol. 302, pp. 350-356, 2016.

[21] B. Qiu, G. Jiang, N. Yang et al., "Discrete element method analysis of impact action between rice particles and impactboard," Transactions of the Chinese Society of Agricultural Engineering, vol. 28, no. 3, pp. 44-49, 2012.

[22] J. Wang, W. Zhou, L. Tian et al., "Virtual simulation analysis and verification of seed-filling mechanism for dipper hill-drop 
precision direct rice seeder," International Journal of Agricultural \& Biological Engineering, vol. 10, no. 6, pp. 77-85, 2017.

[23] X. Wang, S. Zhang, H. Pan, Z. Zheng, Y. Huang, and R. Zhu, "Effect of soil particle size on soil-subsoiler interactions using the discrete element method simulations," Biosystems Engineering, vol. 182, pp. 138-150, 2019.

[24] F. Li, J. Yang, Q. Song, and T. Sun, "Study on discrete element particle flow of multi-layered groundbased expanded bottom uplift pile," Journal of Central South University (Science and Technology), vol. 050, no. 011, pp. 2859-2869, 2019.

[25] R. Xia, B. Li, X. Wang, T. Li, and Z. Yang, "Measurement and calibration of the discrete element parameters of wet bulk coal," Measurement, vol. 142, pp. 84-95, 2019.

[26] Z. Tian, L. Zhao, X. Liu et al., "Loading performance of spiral drum based on discrete element method," Journal of China Coal Society, vol. 10, pp. 2758-2764, 2017.

[27] Y. Li, F. Li, X. Xu et al., "Parameter calibration of wheat flour for discrete element method simulation based on particle scaling," Transactions of the Chinese Society of Agricultural Engineering, vol. 35, no. 116, pp. 320-326, 2019.

[28] F. Liu, J. Zhang, B. Li, and J. Chen, "Calibration of parameters of wheat required in discrete element method simulation based on repose angle of particle heap," Transactions of the Chinese Society for Agricultural Engineering, vol. 32, no. 12, pp. 247-253, 2016.

[29] X. Zhang, J. Zhang, P. Li et al., "Simulation analysis of powder material fluidity," Transactions of the Chinese Society for Agricultural Machinery, vol. 39, no. 8, pp. 196-198, 2008.

[30] F. Peng, H. Wang, F. Fang, and Y. Liu, "Parameter calibration of discrete element model parameters for pellet feed based on injected section method," Transactions of the Chinese Society for Agricultural Machinery, vol. 49, no. 4, pp. 140-147, 2018.

[31] S. Zhang, Z. Shan, and J. Zhang, "Simulation of self-hardening resin sand mobility based on DEM," Foundry Technology, vol. 37, no. 2, pp. 288-291, 2016.

[32] A. Mohammadreza, A. Maryam, G. Mojtaba et al., "A methodology for calibration of DEM input parameters in simulation of segregation of powder mixtures, a special focus on adhesion," Powder Technology, vol. 339, pp. 789-800, 2018.

[33] Q. Zhu, Y. Xing, R. Lu, M. Huang, and P. K. Ng, "Visible/ shortwave near infrared spectroscopy and hyperspectral scattering for determining bulk density and particle size of wheat flour," Journal of Near Infrared Spectroscopy, vol. 25, no. 2, pp. 116-126, 2017.

[34] C. G. Jange and R. P. K. Ambrose, "Effect of surface compositional difference on powder flow properties," Powder Technology, vol. 344, no. 15, pp. 363-372, 2019.

[35] S. Kumar and C. S. Saini, "Study of various characteristics of composite flour prepared from the blend of wheat flour and gorgon nut flour," International Journal of Agriculture, Environment and Biotechnology, vol. 9, no. 4, pp. 679-689, 2016.

[36] R. Bharadwaj, W. R. Ketterhagen, and B. C. Hancock, "Discrete element simulation study of a Freeman powder rheometer," Chemical Engineering Science, vol. 65, no. 21, pp. 5747-5756, 2010.

[37] A. K. Olaleye, O. Shardt, G. M. Walker et al., "Pneumatic conveying of cohesive dairy powder: experiments and CFDDEM simulations," Powder Technology, vol. 357, pp. 193-213, 2010.

[38] A. Andrew, S. McClure, and M. Gentzler, "XRCT characterization of mesoscopic structure in poured and tapped cohesive powders and prediction by DEM," Powder Technology, vol. 330, pp. 386-396, 2018.
[39] P. Stefan, Y. Slavina, J. Alvaro et al., "An experimentally validated DEM study of powder mixing in a paddle blade mixer," Powder Technology, vol. 311, pp. 287-302, 2018. 